(Aus dem physiologischen Institut in Göttingen.)

\title{
Untersuchungen über den Lungenvagus.
}

Von

\author{
Dr. Heinr. Boruttau, \\ Assistenten und Privatdozenten.
}

(Hierzu Tafel I, II und III.)

Die Erscheinungen, welche für die Beziehnngen des N. vagus zur Athmung in Betracht kommen, sind, wie schon lange bekannt, bei verschiedenen Thierarten nicht vollständig die gleichen. Während die meisten, in den letzten Jahren fast alle, hierhergehörigen Untersuchungen an Kaninchen angestellt worden sind und die Ergebnisse derselben grossentheils als feststehend betrachtet werden können, sind bei weitem abweichende und zum Theil einander widersprecbende Erscheinungen sowohl bezïglich der Vagusreizung wie beztiglich der beiderseitigen Vagusdurchschneidung beim $\mathrm{Hu}$ ude erhalten worden. Diese waren es, welche den Ausgangspunkt zahlreicher Versuche bildeten, die ich, grösstentheils in Gemeinschaft mit Herrn cand. med. F ri ck e im Winter 1894/95 an Hunden, Katzen and Kaninchen, sowie einer Ziege angestellt habe. Die Ergebnisse derselben enthielten manche theils neue, theils die weniger bekannten Versuchsresultate anderer Autoren bestätigende Thatsachen und ergaben eine so klare Einsicht in die den in Rede stehenden Thierarten gemeinsame Bedeutung des Lungenvagus, dass trotz der bereits so grossen Menge der Veröffentlichungen auf diesem Gebiete es sich rechtfertigen lassen dürfte, dieselben im Folgenden mitzutheilen. Es sollen dabei erst die durch Vagusreizung zu erzielenden. Wirkungen auf die Athmung besprochen werden, sodann die durch die beiderseitige Vagusdurchschneidung erzeugten Ausfallserscheinungen und Lungenveränderungen. $\mathrm{Da}^{*}$ eine vollständige Zusammenstellung der neueren hierhergehörigen Literatur fehlt und ich durch die vorliegenden Untersuchungen veranlasst wurde, eine solche vorzunehmen, so gebe ich dieselbe am Schlusse wieder, mit dem Bemerken, dass ich nach Möglichkeit 
alle Arbeiten berïcksichtigt habe, welche die Beziehungen des N. vagus zur Athmung entweder selbst behandeln oder näher damit zusammenhängen, wäbrend diejenigen Veröffentlichungen nicht aufgezählt werden konnten, in welchen diese Beziehungen nur beiläufig erwähnt sind.

I.

Die widersprechenden Resultate der fast ausschliesslich an Kaninchen angestellten elektrischen Reizversuche an den centripetalen, reflektorisch die Athmung beeinflussenden Vagusfasern führten bekanntlich zur Berücksichtigung bestimmter, bei diesen Versuchen möglicher Fehlerquellen - Stromschleifen und unipolare Abgleichungen auf den $\mathrm{N}$. laryngeus superior (Rosenthal) und phrenieus (Gad), - sowie zur Aufsuchung von zunächst in den Versuchsthieren selbst gelegenen Faktoren, welche für die Art des Reizeffektes (ob inspiratorisch bezw. beschleunigend oder exspiratorisch bezw. verlangsamend) maassgebend sein sollten: Thierart, Geschlecht, veränderlicher, insbesondere durch Narkotika versehieden beeinflusster Zustand der Centralorgane. Dem gegenüber gebührt $G$ rütz $n \in r^{1}$ ) und Langendorff ${ }^{2}$ ) das Verdienst, zuerst auf die wichtige Bedeutung der Art des Reizes für den auf die Athmung zu erzielenden Effekt hingewiesen und in dieser Richtung. Untersuchungen angestellt zu haben, welche sich anf Anwendung mechaniseher, thermischer und ehemischer Einwirkungen, sowie konstanter Kettenströme, statt der bis dahin allein angewendeten Induktionsströme bezogen. Bevor ich dazu übergehe, die im Anschluss hieran von uns angestellten Versuche und aus denselben zu entwickelnden Vorstellungen zu besprechen, bemerke ich hinsichtlich der vorher erwähnten, in den Versuchsthieren selbst zu suchenden Faktoren, dass wir unbeschadet der Bedeutung der Art des Reizes, die Mitwirkung dieser Faktoren grossentheils haben bestätigen können, indem je $\mathrm{nachder} \mathrm{Art}$ und dem Zustand des Thieres bald die inspiratorischen, bald die exspiratorischen Effekteleichter und a usgesprochener erbalten wurden, jedesmal bei

1) Pflüger's Arch. XVII, S. 250. LVII, S. 98.

2) Mittheilungen aus dem Königsb. physiolog. Labor., S. 50-55. Pflüger's Arch. IIX, S. 201. 
Anwendung derjenigen, weiter unten zu erörternden Art der Reizung, die als solche ausschliesslich inspiratorisch bezw. exspiratorisch wirkt.

Während z. B. bei den meisten $K$ an inchen die inspiratorische Beschlennigung oder der Stillstand der Athemmuskeln in Inspirationsstellung leichter zu erhalten ist, als die exspiratorische Wirkung, fanden wir einzelne Exemplare, bei welchen die inspiratorische Wirkung nur schwer, fast stets dagegen exspiratorische Wirkung: erbalten wurde; auch beobachtete ich, wie Meltzer ${ }^{1}$ ), dass die inspiratorischen Wirkungen bei männlichen, die exspiratorischen bei weiblichen Kaninchen besser ausgesprochen sind. Bei Kat z e n ist die exspiratorische Wirkung regelmässig die leichter zu erzielende, ebenso wie beim $\mathrm{Hu}$ ud, was ausser den Ergebnissen älterer Arbeiten (Arloing und Tripier, P. Bert) auch den ganz neuerdings gemachten Angaben von $\mathrm{Ka}$ a de rs ${ }^{2}$ ) entspricht. Auch wechselte die iuberwiegende Wirkung bei demselben Thiere im Laufe der Versuche, eine Erscheinung, die wohl mit Recht Veranlassung gegeben hat, die in Rede stehenden Unterschiede auf den Zustand der Nervencentra, nicht der Vagusfasern zurückzuführen; für diese Anschaung spricht aber vor allem der Einfluss narkotischer Gifte, besonders des Chlorals. Die Begünstigung der exspiratorischen Wirkungen durch tiefe Chloralnarkose fand ich, wie früher Frederic $q^{3}$ ), Christian $i^{4}$ ) und Langendorffor), sehr ansgesprochen. Dieser Umstand veranlasste uns, die Reizversuche meist an nicht narkotisirten Thieren anzustellen, um die Bedeutung der Art des Reizes möglichst von den andern Faktoren zu sondern ${ }^{6}$ ). Zur sichern Erkenntniss dieser Bedeutung ist

1) Centralbl. f. d. med. Wiss. 1882, S. 497.

2) Pflügers Arch. LVII, S. 333.

3) Bull. de l'acad. de Belgique (2) 47, p. 24.

4) Monatsber. d. Berl. Ak.; 1881, S. 222.

5) du Bois' Arch., 1881, S. 526.

6) In diesem Sinne äussern sich mit Recht Pawlow und Schumowa in einer erst ganz kürzlich erschienenen Arbeit (s. weiter unten S. 113. Anm.) dahin, dass durch die heute allgemein übliche übermässige Anwendung der narkotischen Gifte die za beobachtenden physiologischen Erscheinungen durchweg stark entstellt werden. Ich schliesse mich dem vollständig an und darf auch bemerken, dass im hiesigen Institut die zur Untersuchung und Demon- 
ferner eine Versuchsmethodik nothwendig, welche die Art der W ir k u n g möglichst klar vor Augen führt.

Hierzu sind - wenn man ron der direkten Inspektion ganz absieht - die verschiedenen gebräuchlichen Methoden graphischer Aufzeichnung der Athembewegungen in sehr verschiedenem Maasse geeignet, ein Umstand, dem bisher anscheinend nicht immer die nöthige Beachtung geschenkt wurde.

Diejenigen Methoden, bei welchen die Bewegungen eines bestimmten Muskels oder einer Muskelgruppe aufgezeichnet werden, z. B. des Zwerchfells oder der Thoraxmuskulatur, können kein vollständiges Bild des auf reflektorischem Wege durch die Vagusreizung erzielten Effektes geben, weil Bewegungen der einen Muskeln nicht gleichzeitig mit Bewegungen der anderen stattzufinden brauchen, ja die inspiratorische Wirkung der einen Muskelgruppe selbst durch gleichzeitige Aktivität der antagonistischen Exspirationsmuskeln ganz oder theilweise kompensirt werden kann ${ }^{1}$ ). Der Gesammteffekt aller in jedem Augenblicke auf den Thorax wirkenden bewegenden Kräfte lässt sich nur veranschaulichen durch Verbindung der Athemwege mit einer, die pulmonalen Volum- oder Druckschwankungen registrirenden Vorrichtung.

In dieser Hinsicht ist am gebräuchlichsten die M a rey'sche Schreibkapsel, welche unter Einschaltung eines Luftreservoirs (Bert'sche oder Hering'sche Flasche) mit der Luftröhre des Versuchsthieres verbunden wird. Von dieser Methode haben auch wir gelegentlich Gebrauch gemacht; in der überwiegenden Zahl der an Kaninchen und Katzen angestellten Versuche benutzten wir dagegen den Athemvolumschreiber von Gad. Bei Hunden, für welche ein Volumschreiber von genügenden Dimensionen nicht zur Verfügung stand, registrirten wir entweder die pulmonalen Druckschwankungen, wie erwähnt, mit der Marey'schen

stration der wichtigen Funktionen bestimmten Tbierversuche von jeher, wenn irgend möglich, ohne Narkose angestellt werden.

1) Vergl. übrigens hierüber Rosenthal (du Bois' Arch. 1880, Suppl.Bd., 49 .

Hier sei auch an die Versuche Marckwalds (Zeitschr. f. Biol., XXIII.) erinnert, bei welchen nach Durehschneidung beider Phrenici passive, von

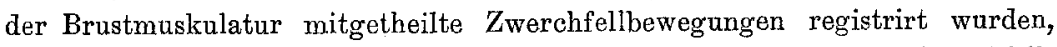
deren Richtung in Bezug auf In- und Exspiration den aktiven Zwerchfellbewegnngen entgegengesetzt war. 
Kapsel, oder die Exkursionen der Thoraxwand durch einen in der Gegend der unteren Apertur um den Thorax gegürteten flachen Beutel aus weichem Kautschuk, der in bekannter Weise mit der Mar ey'schen Kapsel verbunden war ${ }^{1}$ ). Mehrfach nahmen wir mit zwei dieser Methoden gleich zeitig Kurven auf; so zeigt Fig. $4 \mathrm{c}$ auf Tafel I ein Beispiel der gleichzeitig registrirten Athemvolumschwankungen und Thoraxbewegungen beim Kaninchen, Fig. $4 \mathrm{~b}$ die Registrirung der pulmonalen Volumschwankungen gleichzeitig mit den Zwerchfellbewegungen. Die Bedeutung der Beweg u g srichtung ist hierbei in den beiden Kurven die entgegengesetzte, indem je in der Druck- resp. Volumkurve der absteigende Schenkel Inspiration und der aufsteigende Exspiration bedeutet, dagegen in der Thorax- resp. Zwerchfellkurve umgekehrt der aufsteigende Schenkel inspiratorischen, und der absteigende exspiratorischen Sinn hat. Von der durch die Autoren viel benutzten Registrirung der Zwerchfellbewegungen haben wir nur bei einem Versucbsthier Gebrauch gemacht ${ }^{2}$ ), sonst aber deshalb nicht, weil wir die dazu nothwendige, wenn auch kleine Bauchwunde als komplizirenden Eingriff vermeiden wollten, besonders mit Rücksicht auf die Erhaltung und weitere Beobachtung der Thiere nach Durchschneidung beider Vagi.

Bei der Beschreibung der Reizversuche beginnen wir aus Gründen, die sich im Folgenden von selbst ergeben werden, mit der Anwendung der nicht elektrischen Einwirkungen. Hinsichtlich des Erfolges sämmtlicher Arten derselben, der mechanischen, thermischen und chemischen, geben Grützner ${ }^{3}$ ), Langendorff ${ }^{4}$ und $\mathrm{Gad}^{5}$ ) ibereinstimmend an, stets nur exspiratorisebe, die Athmung verlangsamende oder ganz hemmende Wirkungen beobachtet zu haben. Wir können diese Angaben bestätigen hinsichtlich der chemischen Reizung, die wir mit Glycerin, konzentrirter Kochsalzlösung und Alkalilaugen vornahmen, sowie bezuiglich der thermischen Einwirkungen: Applikation sehr heisser

1) Zuerst von Knoll angegebene Vorrichtung: Prager med. Woch., 1879.

2) Sie erfolgte hier durch Luftübertragung der Bewegungen eines improvisirten Zwerchfellhebels.

3) a. a. 0 .

4) Mittheilungen a. d. Königsb. Labor., S. 50-55.

5) du Bois' Arch., 1880, S. 11. 
Flüssigkeit oder glibhenden Metalls (Thermokauter). Dagegen fand ich die Angaben bezïglich ausschliesslich exspiratorischen Erfolges mechanischer Einwirkungen $\mathrm{n}$ i $\mathrm{ch}$ t bestätigt, ebensowenig wie die gegentheilige Angabe von $\mathrm{Knoll}^{1}$ ), dass „der primäre Effekt mechanischer Reizung stets in s pir a to r is ch sei", indem unsere Versuche unzweideutig ein Resultat hatten derart, wie es früher bereits von $\mathrm{Traube}{ }^{2}$ ) erhalten wurde, indem dieser beobachtete, dass ,ein kurzer inspiratorischer Stillstand oder vielmehr eine tiefe Inspiration" eintritt, "wenn man den N. vagus mit der Scheere schnell durchschneidet" (und zwar zunächst den einen Vagus bei erhaltener Kontinuität des andern). Gerade in diesem Fall, be i präziser Durchschneidung mit scharfer Scheere, beobachten auch wir öfters vertiefte Inspiration oder Beschleunigung in Inspirationsstellung - wovon Figur $2^{\text {a }}$ ein Beispiel an der Katze, Fig. 3 am Kaninchen giebt -, während Durchtrennung mit einem s t u m p f e $\mathrm{n}$ Instrument, sowie $\mathrm{D} \mathrm{u} \mathrm{r} \mathrm{c} \mathrm{b-}$ s c h n ür ung im Gegentheil, wie noch genauer erörtert werden wird, exs piratoris che $\mathrm{s}$ Sills tand der Athmung herbeifübrte. Dass bei der inspiratorischen Wirkung des Schnittes es sich um eine Täuschung durch elektrische Reizung infolge Ungleichartigkeit der Scheerenblätter gehandelt habe, wird höchst unwahrscheinlich durch das Ergebniss von Versuchen mit einem, mit Elfenbeinhammer versehenen, elektromagnetisch angetriebenen mechanischen Tetan o motor ${ }^{3}$ ). Die Wirkung der frequenten äusserst kurzen Schläge dieses Instruments auf den undurchschnittenen oder durchschnittenen Vagus war in den meisten Fällen, im Gegensatz zu den Angaben Langendorff's, evidente inspiratorisehe Beschleunigung, wovon in Fig. 1 ein Beispiel, herrührend von einer Katze, gegeben ist ${ }^{4}$ ). Selbstverständlich wurde durch Kontrollversuche ausgeschlossen, dass eine derart ausgesprochene Athemveränderung lediglich auf psychische Erregung des Thieres durch den Lärm des spielenden Instruments zuriekzufïhren gewesen wäre.

1) Berichte der Wien. Akad., math.-nat. Kl., LXXXVI, 3. Abth., S. 58.

2) Gesamm. Beitr. z. Pathol. u. Physiol., 1871, Bd. I, S. 188.

3) Das gleiche gilt für die Täuschung durch die noch zu besprechenden Ausfallerscheinungen.

4) Das gleiche beobachtete Knoll (a. a. 0.), als er den Vagus mit einer schwingenden Darmsaite berührte. 
Gegenüber diesen Wirkungen sehr s chn ell verlaufender mechanischer Einwirkungen fand ich den ausschliesslich exspiratorischen Effekt bestätigt für die Durchschnürung - wovon Fig. $2^{\mathrm{b}}$ ein Beispiel giebt, welches sich bezieht auf Durchschniurung des centralen Stumpfes desselben Vagus, dessen schnelle Durchschneidung vorher die Kurve Fig. $2^{a}$ geliefert hatte - , sowie für danernde Quetschung oder Zerrung, wie sie bei unvorsichtiger Präparation oder zu gewaltsamem Herausholen aus der Wunde vorkommen $k^{2}{ }^{1}$ ).

Indem ịch diesen Arten mechanischer Einwirkung die chemischen und thermischen Applikationen als k ontinuirliche oder Da u er-Einwirkunge ${ }^{2}$ ) zur Seite stelle, glaube ich deshalb für den Erfolg nicht elektrischer Applikationen auf den Vagus aus unseren Versuchsergebnissen den Satz folgern zu dürfen, dass moment an e E i n wirkung e n, einmalig (Schnitt) oder öfter wiederholt (Tetanomotor) ins piratoris c be, dagegen dauernde Einwirkungen exspiratorischen Erfolg hauen.

Durch eine hiermit übereinstimmende Vorstellung erhalten wir auch eine ungezwungene Deutung der Resultate elektrischer Vagusreizung; welche bei der Unsicherheit der Wirkung der fast ausschliesslich angewendeten Induktionsströme bis vor Kurzem von Vielen als jeder Erklärung unzugänglich angesehen wurden. Nachdem bereits vor einer Reihe von Jahren Grützner ${ }^{3}$ ) gefunden batte, dass ein durch den centralen Stumpf des durchschnittenen Vagus in aufsteigender Richtung geleiteter konstanter Kettenstrom regelmässig bei seiner Schliessung und während seiner Dauer exspiratorischen Athmungsstillstand hervorruft, und dass die Oeffnung eines absteigenden Stroms ebenso wirkt, haben ganz neuerdings Langendorff und Oldag ${ }^{4}$ ) ,das Verbalten der die Athmung beeinflussenden Vagusfasern gegen Ketten-

1) Die Betheiligung des Längsquerschnittstromes des Nerven bei diesen Wirkungen wird weiter unten noch zu besprechen sein.

2) Ich vermeide hier absichtlich den Ausdruck "Reizung", da nach den herrschenden Ansichten demselben das Moment des Diskontinuirlichen, der Intensitätsschwankung, innewohnt.

3) Pflügers Arch., XVII, S. 248-49.

4) ibid., LIX, S. $201 \mathrm{ff}$. 
ströme“ zum Gegenstand einer ausführlichen Untersuchung gemacht, welche erschien, als wir unsere Versuche bereits begonnen batten und deren Resultate wir durch ausfübrliche Nachprüfung und Erweiterung, soweit als die Thatsachen in Betracht kommen, vollständig bestätigt fanden.

Zur Zuleitung der Ströme benutzten wir d'Arsonval'sche Chlorsilberelektroden in Hakenform; ïbrigens ist bei der mässigen Stärke der zu verwendenden Ströme Unpolarisirbarkeit der $\mathrm{Zu}$ leitungselektroden nicht unbedingtes Erforderniss, wogegen sebr viel darauf ankommt, dass dieselben sammt dem aufliegenden Nervenstumpf mit der Wunde in keinerlei Berührung stehen; insbesondere sind bei diesen Versuchen tiefliegende Elektroden in Hartgummihiille durchaus zu vermeiden, indem sonst die noch näher zn besprechenden Alterationen der Reizwirkungen durch Stromschleifen eintreten. Zur Erzielung der für die Kettenströme charakteristischen Wirkungen genügen mässige Stromstärken, welchc wir, wie Langendorff durch 2 bis 6 Chromsäure-Elemente unter Einschaltung eines Stöpselrheostaten in Nebenschliessung hervorbrachten.

Wird ein derartiger k onstanter Strom in a u fsteigend e r R i c htung durch das centrale Vagusende geleitet, so erfolgt beträchtliche Verlang sam ung der Athmung bis zum Stillstand in Exspirationsstellung, welcher während der ganzen Durchström ungs-Dauer anhält. Wir konnten dies Verbalten an sämmtlichen von uns benutzten Thierarten konstatiren und wird solches durch Figg. 4, 5 und 6 für Kaninchen, Hund und Katze verdeutlicht ${ }^{1}$ ).

1) Die exspiratorischen Effekte bei allen elektrisehen und nicht elektrischen Einwirkungen bestehen ausschliesslich in Hemmung der Thätigkeit der inspiratorischen Muskeln; aktive Exspiration ist sehr selten, sofern Stromschleifen vermieden werden, und mit Ausnahme des Hundes. Die Häufigkeit der aktiven Exspiration bei dieser Thierart wird bei den Ausfallserscheinungen noch näber erörtert werden.

Einen besondern Fall der Wirkung aufsteigender Durchströmung bilden die exspiratorischen Effekte durch Nebenschliessung des Längsquerschnittsstromes ("Demarkationsstromes") des durchschnittenen Vagus durch die feuchten Gewebe, auf welche Knoll (a. a. O.) durch ausführliche Versuche mit Recht die Aufmerksamkeit gelenkt hat. Dieselben können die inspiratorische Wirkung der Durchschneidung verdecken, wenn der Stumpf in die Wunde fällt. (Vergl. Gad, Lehrb., S. 411). 
Durehströmung des centralen Vagusstumpfes durcb einen absteigenden Strom ist oft ohne Wirkung; tritt eine solche ein, so besteht sie in einer vertieften, bez. verlängerten Inspiration im Augenblicke der $\mathrm{Schli}$ es s u g, die noch einigemal sich wiederbolen kann, nicht aber während der ganzen Durchströmungsdauer (Fig. 7). Die $\mathrm{O}$ e f f $\mathrm{n} \mathfrak{u} \mathrm{n}$ (bez. Abblendung durch Kurzschluss) des a u s teige $\mathrm{n}$ den Stromes erzengt oft inspirat 0 ris ebe (Fig. 8, 9, 10) und diejenige des absteig enden Stromes regelmässig exspiratorische Wirkung (Fig. 7), die sich durch das nummehr alleinige Bestehen des polarisatorischen Nachstroms am einfachsten erklärt, welcher bekannntlich im Nerven, wie in jedem zersetzbaren Leiter dem polarisirenden Strome entgegengesetzt gerichtet ist.

Im Anschluss an die Wirkungen des konstanten Stromes angestellte Versuche mit unterb rochenen Kettenströmen hatten L angendorff und Oldag das Ergebniss geliefert, dass absteigend gerichteten unterbrochenen Kettenströmen unter allen Umständen inspiratorische Wirkung zukommt, dass aber dasselbe auch bei anfsteigenden unterbrochenen Strömen der Fall ist, wenn dieselben frequent und von kurzer Schliessungsdauer sind, sowie wenn das Versuchsthier (Kaninchen) nicht oder nur schwach (mit Chloralhydrat) narkotisirt ist. Dagegen erhielten sie exspiratoris che. Wirkung, wenn die Frequenz der Unterbrechung des aufsteigenden Stromes sehr gering und die Schliessungsda e r im Verhältniss zur Unterbrechungsdauer gross war, sowie bei tiefer Chloralnarkose. Von diesem letzten Punkte, der zu den anfangs besprochenen Faktoren gehört, ganz abgesehen, ersieht man leicht, dass diese Thatsachen, die wir vollständig bestätigt fanden s. Fig. 11-14 - darauf hinausgehen, dass Stro ms tös s e ins piratorisch, dauernde Durchströmung bei a ufsteigender Richtung exspiratorisch wirken, worin sofort die Analogie zu den Effekten der nichtelektrischen Einwirkungen sich zeigt. Zur b e l i e b i g fre q $\mathrm{u}$ e $\mathrm{n}$ t e n Unterbrechung der Kettenströme dienten uns die schon bei einer anderen Gelegenkeit ${ }^{1}$ ) erwähnten, als a $\mathrm{u}$ o mat is che $\mathrm{W}$ ip p e $\mathrm{n}$ bezeichneten

1) Pflüger's Archiv, LVIII, S. 15. 
Apparate, welche bei gekreuzter Drahtverbindung uns auch zu den weiter unten za besprechenden Versuchen mit Wechselströmen dienten, und von denen der eine durch vertikale Verstellbarkeit der Quecksilbernäpfe die Möglichkeit bot, das Verhältniss der Sehliessungs- zur Unterbrechungszeit beliebig zu verändern.

Indem Langendorff seine Versuchsergebnisse so deutet, dass, gleichgültig ob konstant oder unterbrochen, au f s t e i g e nde Strömeexspiratorisch und absteigende inspirat or i s ch wirken sollen, ist er genöthigt, unter der gleichzeitigen Annahme, dass beide Wirkungen von derselben Elektrode, nämlich der Kathode ausgehen, „der dem Centralorgan näheren Elektrode eine bemmende Wirkung, der entfernteren ein tetanisirendes Element" zuzuschreiben, wofür ein Analogon in der ganzen Muskelund Nervenphysiologie nicht vorliegt. Ueberdies sprechen seine Versuche mit gleichzeitiger und entgegengesetzt gerichteter Durchströmung beider centraler Vagusstümpfe vielmehr für die von uns oben angedeutete Erklärung, indem er in denselben bei konstantem Strom stets exspiratorische, bei unterbrochenem Strom stets inspiratorische Wirkung erhielt.

Unsere Vorstellung liess zunächst nur die eine Frage offen, warum beim a $\mathrm{f}$ s teige $\mathrm{n}$ de $\mathrm{n}$ konstanten Strom dessen Da uer, beim absteigenden $\mathfrak{n} \mathfrak{u}$ der Moment der Schliessung wi r k t. Das nächstliegende war, da die Versuche Langendorff's und die, eine Wiederholung derselben bildenden unserigen am durchschnittenen Vagus angestellt waren, an eine Wirkung des Querschnitts zu denken. Reizversuche, welche wir nach übrigens gleicher Methode am undurehschnittenen Nerven ausführten, hatten nun das bemerkenswerth erscheinende Ergebniss, dass die Bedeutung der Stromrichtung für den Reizerfolg genau die umgekehrte wurde, wie bei Durchströmung des centralen Stumpfes. Vgl. Fig. 15-17.

Die Erklärung hierfür ergab sich indessen bald aus der von Rosenthal ${ }^{1}$ ) betonten Fehlerquelle, die beim undurchschnittenen, central und peripherisch mit dem Versuchsthier zusammenhängenden Nerven darin liegt, dass der durch zwei Elektroden zugeführte Strom entsprechend 'den Kirchhoff'schen Gesetzen sich verzweigt, indem ein Antheil die intrapolare Strecke durch-

1) du Bois' Arch., 1881, S. $62 \mathrm{ff}$. 
läuft, ein anderer Zweig aber durch die extrapolaren Nervenstrecken und den Körper des Thieres seinen Weg nimmt, also für den Nerven in entgegengesetzter Richtung, wie der intrapolare $Z$ weig. Ist nun die extrapolare Streckenlänge im Verhältniss zur intrapolaren nicht besonders gross, wie es bei Versuchen an kleineren Thieren meistens der Fall ist, berücksichtigt man ausserdem, dass der "fehlerhafte Stromzweig" in dem Thierkörper auch das Centralorga n durchfliesst, so ist, die verkehrte Wirkung ohne weiteres verständlich. Die Richtigkeit dieses Sachverhalts ergab sich übrigens noch durch Versuche, in welchen der Nerv erst undurchschnitten gereizt wurde (Fig. 18), dann peripherisch von den Elektroden durchschnitten und wieder gereizt wurde (Fig. $19 a$ bis $c$ ), wobei die Wirkung sich umkehrte, und endlich der über der peripherischen Elektrode herabhängende Stumpf wieder in leitende Berührung mit der Wunde gebracht wurde: jedesmal, wenn während der Dauer dieser Beriuhrung der Reizstrom applizirt wurde, zeigte sich die Wirkung (Fig. 20) der von Rosenthal so bezeichneten "falschen Nervenreizung", zu welcher übrigens noch Vermischung der Wirkung mit Schmerzäusserungen trat, wie denn endlich zur Mitreizung anderer Nerven in diesem Falle Gelegenheit gegeben ist. Es beweisen diese Versuche jedenfalls a fortiori, dass dem aufsteigenden Strom als solehen die exspiratorisch-hemmende Dauerwirkung und dem absteigenden die inspiratorisch-erregende Schliessungswirkung zukommt. Zur Erklärung suchten wir nun diese Thaț̦ache mit bekannten Sätzen der allgemeinen Nervenphysiologie, sowie mit den von mir anlässlich der Untersuchungen über das galvanische Erregungsphänomen des Nerven gemachten Erfahrungen in Verbindung zu bringen, indem wir uns fragten, von welcher Elektrodedie Wirkungen a s gehen, und anf welchen in der allgemeinen Naturder Nervenfaserbe. grïndeten $T h a t s a c h e n$ sie beruhen.

Die Ausdehnung der anf Grund gewisser Thatsachen der allgemeinen Muskelphysiologie von einigen Autoren gemachten Annahme, dass entsprechend der von der Kathode ausgehenden Erregungswirkung von der Anode aus eine Hemmungs wirkung in 'erregbaren Geweben sich ausbreite, die Ausdehnung dieser Annahme auf den uns vorliegenden Fall erschien aus mehreren Gründen unthunlich: einmal, weil es sich hier um reflektorisch durch die uns unbekannten Vorgänge im Centralorgan vermittelte 
Wirkungen handelt, hauptsächlich aber, weil bei dem, eben gerade exspiratorisch wirkenden a u f teigenden Strom die A node die vom Centralorgan entferntere Elektrode ist, beim a bste igende n Strom dagegen, wo die Anode dem Centralorgan zunächst liegt, während der Durchströmung k e i n e He m mu ng swirkungen auftreten. Diese Thatsachen weisen vielmehr darauf hin, dass, wie auch Langend orff annimmt, beide Arten der Wirkung von demselben Pole, nämlich der K a th od e, a usge he n. Die Richtigkeit dieser Vorstellung noch besonders za beweisen gelang uns mit Hülfe der sonst im allgemeinen wegen ihrer Fehlerquellen in der Physiologie zu vermeidenden Methode, welche darin besteht, dass nur eine Elektrode dem undurchschnittenen Nerven angelegt wird, während der andere Pol der Kette durch eine plattenförmige Elektrode von grosser Oberfläche mit der wohlbefeuchteten Haut des Thieres verbunden wird: da, dem kleinen Querschnitt entsprechend, die Stromdichte an der einen, dem Nerven ànliegenden Elektrode unvergleichlich grösser ist, als an der anderen, der Körperoberfläche anliegenden, so kommt fast nur die Wirkung der ersteren in Betracht, die deshalb in der Elektrotherapie auch als "differente" Elektrode im Gegensatz zu der ,,indifferenten“ Plattenelektrode bezeichnet wird. Es zeigte sich nun eine deutliche exspiratorische Wirkung der and a u ernden·Durehströmung nurdann, wenn die differente Elektrode Kathode war, während jede Wirkung fehlte, wenn sie zur Anode gemacht wurde (Beispiel s. Fig. 21 $a, b)$. Wurde der Nerv peripherisch von der „differenten" Elektrode durchschnitten, so trat sofort das umgekehrte ein, indem eben im ersten Fall der centrale Nervenstumpf absteigend, im zweiten Fall aufsteigend durchströmt war ${ }^{1}$ ). (Fig. 22.) Versuche, die Bedentung der beiden Elektroden durch wirklich „unipolare" Applikation festzustellen, indem der eine Pol der Kette dem Nerven des durch Hartkautschukunterlage vollständig isolirten Thieres angelegt, der andere Pol mit der Erde verbunden wurde, blieben bei der von uns benutzten Anzahl der galvanischen Elemente ohne jedes Ergebniss.

Nachdem immerhin die Bedeutung der Kathode als aus-

1) Nach dieser Methode führten Langendorff und Oldag einen Theil ihrer Versuche aus. (a. a. 0., S. 205). 
sćhliesslich wirksamer Pol ausser jeden Zweifel gestellt ist, lassen die in Rede stehenden Thatsachen nur die eine Deutung zu, dass die während der ganzen Dauer der Durehströmung von der Kathode ausgehenden Wirkungen es sind, welche, bis zum Centralorgan reichend, die ex spiratorischen Effekte veranlassen, dass dagegen der im A g enblick e des Stromschlusses von der Kathode ausgehende wellenförmige elektrische Vorgang es ist, welcher die inspiratorische Wirkung hervorbringt. Mit anderen Worten: die beim "Entstehen des Katelektrotonus" auftretende katelektrotonische Welle oder „Negativitätswelle“ wirkt inspiratorisch ${ }^{1}$ ), der während der Dauerder Durchströ mung sich extrapolar ausbreitende ,ka telektrotonische Strom" exspiratorisch.

So wird es erklärlich, warum einigermaassen frequent unterbrochene und $k \mathfrak{u} z d a \mathfrak{u}$ rnde a u fosteigende Ströme i n s p i r a t o r i s c h wirken; zur Erklärung der gleichen Wirkung der utterbrochenen absteigenden Ströme, sowie der Schliessung des konstanten absteigenden Stromes sei daran erinnert, dass, wie ich gezeigt habe ${ }^{2}$ ), die von der Kathode ausgehende $\mathrm{Negativitäs}$ welle sich $\mathrm{sehneller}$ fortpflanzt bezw. früher entsteht, als die von der Anode ausgehenden extrapolaren Wirkungen; es wird somit bei den absteigenden unterbrochenen Strömen, dieser, inspiratorisch wirkende Vorgang jedesmal vor Entstehen des Anelektrotonus , $\mathfrak{u}$ i ter der A n ode durchgehen", wie ich mich ausdritckte, und auf das Centralorgan wirken. Beim absteigenden constanten Strom ist es nur e in e von der Kathode ausgehende Negativitätswelle, die im Moment der Schliessung unter der Anode weg nach dem Centrum läuft. Dass aufsteigende unterbrochene Ströme auch exspiratorisch wirken können, besonders wenn die jedesmalige Schliessungsdauer relativ lang ist, leuchtet ein, indem das $M o \mathrm{~m}$ e $\mathbf{u t}$ der katelektrotonischen, ,festen Polarisation" über dasjenige der ,Negativitätswellen" die 0 berband gewinnt. Dass

1) Wir können anschliessend an das von Langendorff der vom Centralorgann entfernteren Elektrode zugeschriebene "tetanisirende Element" sagen: sie wirkt „reflektorisch tetanisirend".

2) a. a. O. S. 45. 
ceteris paribus in diesem Falle bei verschiedenen Thieren und in verschiedenem Zustande derselben (Narkose) bald die eine, bald die andere Wirkung erhalten werden kann, erklärt sich ebenfalls leicht aus dem Zusammenwirken der beiden in Rede stehenden Momente mit den in Art und Zustand des Thieres gegebenen Faktoren, welche bald dem einen, bald dem andern Moment zum Ueberwiegèn verhelfen. Genau das gleiche gilt endlich für den in bekannter Weise wechselnden Erfolg der induzirten W e c b s e lstr ö me, wozu, wenn dieselben durch ein Schlitteninduktorium mit Wagner'schem Hammer erzeugt werden, noch ihre Inkongruenz und unbeherrschbare Ungleichnässigkeit kommt. Die kongruenten Wechselströme des Sinusinduktors, sowie kurze, frequente, durch Vermittlung der "automatisehen Wippe“ die Richtung wechselnde Kettenströme ergaben mir durchgehends inspiratorische Wirkungen, wovon Fig. 23 und 24 am Kaninchen gewonnene Beispiele zeigen. Für gleichgerichtete Induktionsströme gilt, wie selbstverständlich, dasselbe, wie für kurze frequente Kettenströme đer entsprechenden Richtung ${ }^{1}$ ). Einzelne Induktionsschläge orgaben bei mässigen Stärken, wie auch Langendorff angiebt, gar keine, oder aber kurzdauernd inspiratorische Wirkung. Die Angaben von We denskii ${ }^{2}$, welcher mit solchen einzelnen Induktionsschlägen reizte, iuber die Bedeutung der $\mathrm{P}$ b ase der $\mathrm{n}$ at ü r lichen A thm ung für de n Re i ze r fol g konnte ich nicht bestätigen.

Die Bedeutung unserer Versuchsergebnisse scheint mir darin zu liegen, dass sie einerseits durch die Analogie der Effekte elektrischer und nicht elektriseher Reizung die allgemeine Anschaung zu stützen geignet sind, dass das Wesen der Nerventhätigkeit in den aus der Struktur der Nerven als K e r n le iter erklärbaren elektrischen Erscheinungen beruht, und dass sie andererseits aus allgemein nervenphysiologischen Gesichtspunkten die anscheinend so vieldeutigen Effekte der Vagusreizung auf die Athmung zu erklären vermögen, und zwar ohne die Nothwendigkeit, zwei verschiedene Faserkategorien für die inspiratorischen und die exspi-

1) Knoll (a. a. 0.) betonte bereits die Bedeutung der Richtung gleichgerichteter Induktionsströme unter Hinweis auf Grützner's Mittheilungen, aber ohne selbst etwas über die Bedeutung der Richtung für die Art des Reizesfolges auszusagen.

1) Pflüger's Archiv, XXVII, S. 1-21. 
ratorischen Wirkungen anzunehmen. A uf die Vorstellungen, welehe über die, diesen Erscheinungen zu Grunde liegenden Vorgänge in den Centralorga $\mathrm{n}$ e $\mathbf{n}$ gebildet werden können, verzichteten wir einzugehen, indem hieraus die Nothwendigkeit erwachsen würde, auf die so zahlreichen Arbeiten über die Athemcentren literarisch und experimentell zurückzugreifen, was die Grenzen unserer Aufgabe zu sehr überschreiten würde. $\mathrm{Zu}$ dieser Beschränkung glaubten wir um so mehr berechtigt zu sein, als auf Grund der von uns gebildeten Vorstellung auch die Untersuchung der bei der normalen Respiration stattfindenden Funktionen des Vagus genïgend ermöglicht erschien.

In dieser Beziehung sei erinnert an die bekannten Versuche von Hering und $\mathrm{Breuer}{ }^{1}$ ), welche zu dem Ergebniss führten, das Aufblasung der Lunge exspiratorisch, Zusammenfallen derselben inspiratorisch wirkt, so lange die Nn. vagi erhalten sind, worauf diese Autoren ibre Theorie von der sog. Selbststeuerung der Athmung durch den N. vagus begründeten. Wir dürfen nunmehr wohl um so eher mit Hering und Breuer die Dehnung der Vagusfasern bei der künstlichen Lungenaufblasung nnd der natiirlichen Inspiration als ein exspiratorisch wirkendes Moment auffassen, als wir diesen exspiratorischen Effect allgemein den mechanischen Dauer-Einwirkungen auf den Vagusstamm (Dehnung, dauernde Kompression) folgen sahen, an welche die Dehnung der in der Lunge sich verzweigenden Vagusfasern sich doch eben anschliesst.

Wir hatten oft Gelegenheit, die exspiratorische Wirkung, resp. Athempause nach Aufblasung der Lange oder blossem Verschluss der eingelegten Trachealkanuile auf der Höhe der Inspiration selbst zu bestätigen, ebenso auch die inspiratorische Wirkung des Zusammenfallens der Lungen bei Eröffnung der Pleurahöhlen. Worin in diesem Falle das inspirationserregende, "tetanisirende"6 Moment zu suchen sei, darüber lässt sich wohl kaum schon etwas Bestimmtes aussagen; dagegen müssen wir auf Grund dẻr jetzt zu besprechenden Versuche mit beiderseitiger Vagusdurchtrennung behaupten, dass beide Momente, das exspiratorisch-hemmende der Lungendehnung und das inspiratorisch wirkende

1) Ber. der Wien. Akad., math.-nat. Kl., Bd. LVII, 2. Ath., S. 672 ff., Bd. LVIII, 2. Abth., S. 909 ff., 1868. 
der Lungenschrumpfung im Sinne der Hering-B re u e r'schen Theorie regulirend, ,steuernd“, bei der normalen Athmung betheiligt sind, wobei wiederum je nach Art und Zustand des Thieres das eine oderdas andere Moment überwiegen kann, indem je nach diesen Faktoren das Gesammtbild der Erscheinungen nach Ausschaltung beider Vagi verschieden ist. Diese Auschaung stimmt iberein mit den ganz neuerdings von $\mathrm{S}$ e b if ${ }^{1}$ ) entwickelten. Vorstellungen.

\section{II.}

Wie schon früher $\mathrm{Br}$ e e $\mathrm{r}^{2}$ ) und $\mathrm{Gad} \mathrm{d}^{3}$, und neuerdings wieder Schiff ${ }^{4}$ ) hervorgehoben haben, ist zur Erkenntniss der Bedeutung des Vagus für die normale Athmung die Ergänzung der Reizversuche durch die Erforschung der bei Vaguslähmung zu beobachtenden A u sfalls ers cheinungen unbedingt nothwendig, und zwar unter Aussehliessung komplizirender Nebenumstände, zu welchen vor allen die $R$ e iz $u \mathbf{n g} \quad \mathbf{d u r} \mathrm{ch}$ den Akt der D u r c trennung selbst gehört. Die Annahme von $\mathrm{Kohts}$ und $\mathrm{T}$ i e g e ${ }^{5}$ ), dass die, besonders beim Hunde schon lange bekannte, auf doppelte Vagusdurchschneidung eintretende starke Verlangsamung der Athmung, verbunden mit mehr oder weniger ansgesprochenen Athempausen, a u s s c h li e s s$1 \mathrm{i}$ ch durch den mit der Durchtrennung verbundenen $\mathrm{Re} i \mathrm{z}$ verursacht sei, erscheint schon desshalb unberechtigt, weil, wie wir sahen, die Durchtrennung, als Sehnitt rase h un d präzise ausgeführt, ins piratoris ch wirkt. An eine danernde Reizung durch den Längsquerschnitstrom des in der Wunde liegenden Nerven ist darum nicht zu denken, weil der durehschnittene Nerv diese elektromotorische Eigenschaft jedenfalls in kürzerer Zeit verliert, als der bisher beobachteten Dauer der verlangsamten Athmung bei lange am Leben erhaltenen, beiderseitig vagotomirten Hunden entspricht. Auf die Angaben, dass in diesen

1) Gesamm. Beitr. zur Physiologie, Bd. I, S. 74.

2) a. a. $0 .$, S. 672 .

3) du Bois' Archiv, 1880, S. 9.

4) a. a. O., S. 99.

5) Pfläg er's Archiv, XIII, S. 87-93. 
Fällen bisweilen die Athmung wieder normale Frequenz erreicht habe, werde ich weiter unten zuriickkommen.

Zur Ausschliessung nun'aller Reizerscheinungen bei der Durchtrennung hat $\mathrm{Gad}^{1}$ ) sich der Aufhebung der Leitung in den $\mathrm{Nn}$. vagi durch Abkühlung derselben unter dem Gefrierpunkt bedient. Dass dabei in der That keine Reizung stattfindet, ist sowohl durch Gad selbst, wie auch durch Knoll'2) bewiesen, welche die Applikation von Kälte auf den centralen Vagusstumpf ohne jede Wirkung auf die Athmung bleiben sahen. Dass die Ausschaltung der normalen Funktionen des Vagus auf die Athmung eine vollständige ist, erhellt daraus, dass Durchschneidung der Vagi peripher von der abgekühlten Stelle nichts weiter an der Athmungskurve ändert. Dieses letztere ist nun aber nach unseren Erfahrungen bereits dann der Fall, wenn die Abkühlung des Nerven bei weitem nicht den Gefrierpunkt erreicht; eine Thatsache, welche, und zwar auch speziell für den Vagus, neuerdings schon von Howel1 ${ }^{3}$ ) gefunden wurde, und mit der älteren Angabe Grütz$n$ e r's ${ }^{4}$ ) übereinstimmt, dass ganz allgemein sowohl die centrifugalen wie cèntripetalen Warmblïternerven bereits zwischen 0 und $+6^{0}$ keinen Reizeffekt an ibren Erfolgsorganen mehr hervorrufen. Dieser Umstand ist aber noch in einer anderen Beziehung bedentungsvoll, insofern nämlich die Wiederherstellung der Leitung durch Wiedererwärmen des Nerven um so schwieriger und unsicherer gelingt, je stärker der Nerv abgekü̈hlt war. Während diese Wiederherstellung der Leitung durch Aufthauen beim hartgefrorenen Nerven bisweilen misslang, vermochten wir die Regulirung der Athmung durch die Vagi vermittelst mässiger Abkühlung bei allen drei Thierarten, Hunden, Kaninchen und Katzen drei bis viermal hintereinander auszuschalten und wiederherzustellen ${ }^{5}$ ). Auf die Grundlagen dieser Erscheinungen näher einzugehen verzichte ich hier, wir sind mit weiteren Untersuchungen darïber beschäftigt.

1) a. a. 0., S. $12 \mathrm{ff}$.

2) Wien. Acad.-Ber., LXXXVI, 3. Abth., S. 62.

3) Journ. of. Physiol., XVI, 298-318.

4) a. a. O., S. 237.

5) Marckwald (a. a. 0., S. 168), welcher die Gad'sche Methodik auf die Ausschaltung des (motorischen) Phrenicus übertrug, giebt an, dass die Wiederherstellung der Leitung nur dann gelang, wenn dieser Nerv nicht stärker als $-2^{0}$ abgekühlt war. 
Die Veränderunge n der Athmung nun, welche auf die reizlose Ausschaltung beider Vagi nach der in Rede stehenden Methode folgen, sind bisher nur am Kaninchen genauer beobachtet, indem Gad darüber folgendes angiebt: Unmittelbar nach dem Auflegen des zweiten Vagus auf den abgektihlten Draht ist die nächste Einathmung stark verlängert und vertieft; und das gleichegill von allen weiteren Inspirationen, so dass entweder geradezu Stillstand in Inspirationsstellung, oder Verlängerung der inspiratorischen Phase. bei jedem Athemzuge an der Athem-Volumkurve zu erkennen ist ${ }^{1}$ ), wobei die nach unten gerichteten inspiratorischen "Thäler" ti efer liegen als vor dem Eingriff; dagegen er reichen di e inspiratorischen, nacho,ben gerichteten Zacken nach Angabe Gad's nicht mehr das frühere Niveau, wobei anch die Dauer der exspiratorischen Phase verkürzt sein kann. Es findet also eine Verschiebung der ganzen A themvolumkurvenach unten, somit eine ,Verschiebung der respiratorischen Mittellage des Thorax im inspiratorischen Sinne" statt. ${ }^{2}$ ) Gad deutet diese Erscheinung dahin, dass durch die Aasschaltung beider Vagi nur der hemmende Einflus s, den die Lungendehnung bei jeder normalen Inspiration durch Vermittlung der Vagi ausibe, in Fortfall komme, so dass jede Inspiration so lange dauere, bis sie dureh, nicht näher angegebene, in den Centren selbst entstandene Einflüsse unterbrochen werde. Diese Unterbrechung sei aber nur von kurzer Dauer, und werde früher, als in der Norm durch den Erregungszustand des Inspirationscentrums überwunden, wie aus der Kurve zu ersehen sei. Dagegen le ug net Gad auf Grund derselben, dass bei der normalen Athmung inspirationserregende Wir$\mathrm{k} u n g e n$ den Centren durch die Vagi übermittelt werden, da sonst nach Fortfall derselben die exspiratorischen Phasen verlängert, statt verkiurzt sein, und die exspiratorischen Gipfel das normale Niveau erreichen müssten.

Wir fanden in unseren zunächst an $\mathrm{Kaninchen} \mathrm{unter}$

1) "absolute" oder "relative inspiratorische Athempause" nach Gad's Bezeichnungsweise.

2) Schiff (a. a. O.) bezeichnet zweckmässig den Vorgang als "Vergrösserung des Werthes Residualluft + Reserveluft". 
genauer Einhaltung der. Gad'schen Methodik ausgeführten Versuchen' (siehe Fig. 25-27), die Verti efung und Verlängerung der Einathmungen vollkommen bestätigt; dagegen sahen wir die exspiratorischen Phasen nur kurze $Z$ eit nach der Durchfrierung gegen die Norm verflacht und ver$\mathrm{k}$ ï rzt; vielmehr erreichten die nach oben gerichteten exspiratorischen Zacken früher oder später stets die gleiche Höhe, wie vor der Durchfrierung der Vagi, und die Dauer der exspiratorischen Phasen war die gleiche wie vorher, oder selbst noch grösser, so dass exspiratorische Pausen zu erkennen waren ${ }^{1}$ ). Obgleich wir also die „Verschiebung der respiratorischen Mittellage im inspiratorischen Sinne" bestätigen können, so müssen wir doch, gegenüber $\mathrm{G}$ ad, aus unseren Kurven schliessen, dass bei der normalen Athmung sowohl die Thätigkeit der Inspirationsmuskeln hemmende als auch inspirationserregende Einflüsse dem (inspiratorischen) Athemcentrum durch die No. vagi zugeleitet werden, a us deren Fortfall nach der "reizlosen Ausschaltung" sich das gleichzeitige A uftreten verlängerter Inspirationen und exspiratorischer Pausen erklärt. Immerhin ist die Thatsache höchst bemerkenswerth, dass je $\mathrm{n}$ ach der Individualität die $\mathrm{Kur}$ ve bei Vagusausschaltung sich bei verschiedenen Thieren derselben Art $\mathrm{nicht} \nabla 0 ̈ 11 \mathrm{ig} g \mathrm{leich}$ gestaltet, woraus folgt, dass auch bei der normalen Athm ung die dem In. spirationscentrum durch die Vagi zugeleiteten, einerseits hemmenden und andererseits anregenden Wirkungen schon bei verschiedenen Individuen derselben Thierart (Kaninchen) in verschiedenem Zustande und verschiedenem Maasse überwiegen ${ }^{2}$ ). In noch höherem Grade zeigt sich diese Verschiedenheit bei ve r-

1) Dabei hatten wir Reizung durch Zerrung u. s. w. aufs sorgfältigste ausgeschlossen. Durchschneidung der Vagi peripher von den abgekühlten Stellen änderte auch nicht das geringste an der Kurve, gleichgültig ob die Abkühlung unter $0^{0}$ erfolgt war oder nicht.

2) Hierdurch möchte ich auch den Unterschied zwischen Gad's und meinen Beobachtungen erklären, zwischen denen diejenigen von Lindhagen (Skand. Arch. f. Physiol. IV) in der Mitte stehen. Ohne Zweifel spielen auch bei der Gestaltung der Ausfallserscheinungen etwa angewendete Narkotika eine Rolle, so das Chloralhydrat, welches schon beim nicht vagotomirten Kaninchen exspiratorische Pausen macht (Gad a. a. O. S. 6). 
sehiedenen Thierarten, wie aus den nunmehr zu beschreibenden, von uns an $\mathrm{Kat} \mathrm{z}$ e $\mathrm{n}$ und $\mathrm{H} \mathrm{u} \mathrm{nd}$ e $\mathrm{n}$ bei ,reizloser Vagusausschaltung" erbaltenen Ergebnissen folgt.

Hierbei ist indessen nocb ein wichtiger, bereits weiter oben angedeuteter Umstand zu beachten, welcher bis jetat vielleicht nirgends genügend berücksichtigt wurde. Es ist dies die a k t i ve Betheiligung der exspiratorischen Muskeln an der Phase der Ausathmung, welche wir in Uebereinstimmung mit mehreren Autoren auf die Thätigkeit eines rom inspiratorischen Athemcentrum besonders za unterscheidenden Exspirationscentrums zurückführen möchten.

Das Auftreten ,aktiver Exspirationen", welches in der Kurve bauptsächjich daran kenntlich ist, dass die exspiratorischen Zacken das normale Niveau (und zwar bedeutend) übersteigen, ist von Sigmand Mayer ${ }^{1}$ ) als bezeichnend fïr den Athmungstypus nach bilateraler Vagotomie hingestellt worden. Nach unseren, sowohl aus der Betrachtung der Kurven, wie auch aus sorgfältiger Inspektion der Thiere und Berücksichtigung des Athemgeräusches geschöpften Erfahrungen, müssen wir das A uftreten aktiver Betheiligung der Exspirationsmuskeln, nach wie vor bilateraler Vagusausschaltung, als s e l t e n e s Vorkommniss bezeichnen beim $\mathrm{Kaninchen,} \mathrm{dagegen} \mathrm{als,} \mathrm{fast} \mathrm{regel-}$ mä s si ges bei dem, später zu besprechenden $\mathrm{H}$ u $\mathrm{n}$ de, endlich als ziemlich häufiges bei der Katze.

B e $i$ di e s e m T h i e r ergab die, wie beim Kaninchen dureh Auflegen auf abgekühlte Drähte bewirkte reizlose Ausschaltung der Vagi eine sofortige und bleibende Vertiefung und mässige Verlängerung. der inspiratorischen Phasen; wirkliche inspiratorische Pausen habe ich bei der Katze nur selten gesehen, öfters aber exspiratorische. Die exspiratorischen Kurvengipfel blieben, wie beim Kaninchen, nur kurze Zeit unter dem normalen Nivean, um dasselbe bald zu erreichen und öfters z a ïbersteigen (Figg. 28 und 29).

In diesen Fällen war denn auch die aktive Betheiligung der Exspirationsmuskeln bei Inspektion der Athembewegungen evident. Der Athemtypus nach beiderseitiger Vagusausschaltung besteht somit bei der $\mathrm{Katze}$ in einer

1) Hermann's Handb. II, 1, S. 271 . 
Verlangsamung der Athmung durch Verlänge$\mathrm{rung}$ beider Phasen, was auf den A us fall in der Norm stattfindender, sowohl eregender, als hemmender $\mathrm{E} i \mathrm{nflüsse}$ anf das Inspirationscentrum hindeutet. Dabei wird die gegen die Norm verspäte te Ausathmung unterstützt und verstärkt durch aktive Betbeiligung der Exspirationsm skeln.

Noch regelmässiger findet diese statt, und noch etwas anders als bei Kaninchen und Katze gestalten sich die Ausfallserseheinungen nach beiderseitiger Vagusdurchtrennung beim $\mathrm{H} \mathrm{und}$. In den meisten an diesem Thiere angestellten Versuchen isolirten wir vor der Durchtrennung jeden Vagus von dem mit ihm in gemeinschaftlicher Scheide verlaufenden Sympathicus nach Eröffnung derselben und vermieden durch diese meist leicht gelingende Maassregel vollständig die durch Sympathicusdurchschneidung: bewirkten Erscheinungen ${ }^{1}$ ).

In einer Versuchsreihe nun, bei welcher wir an Hunden beide Vagi, besonders zum Studium der Lungenveränderungen, einfach mit der Scheere durchschnitten hatten, sahen wir, nach Vorübergehen der Reizsymptome, die Athemfrequenz bis nahe zur Hälfte vermindert, die Inspirationsdauer verlängert, die Exspirationen aktiv und zwischen den einzelnen Athemzügen oft sehr lange Athempausen, in denen die inspiratorisehe Athemmuskulatur anscheine nd ganz in Ruhe ist. Die graphische Aufnahme nach dem Bert'schen Verfahren (das genauere siehe weiter unten), ja schon die genauere Untersuchung durch Inspektion und Palpation ergiebt indessen, dass nur der Anfang der Pausen rein exspiratorischer Natur ist, indem ganz allmählich und langsam, erst unmerklich, dann immer kräftiger und deutlicher, die inspiratorischen

1) Bisweilen verläuft der Vagus eine Strecke weit getrennt vom Sympathicus. Einem derart isolirten Verlauf nur eines Theils der Vagusfasern sind wir nie begegnet und können somit die Angabe von Jolyet (Gaz. médicale de Paris, 1873, S. 37) nicht bestätigen, welcher neben dem linken Vagus einen besondern Faden fand, dessen Reizung auf die Athmung (speciell hustenerregend) wirkte. Uebrigens ist die Isolirung des Vagus vom Sympathicus nie ganz ohne Auftreten von Reizerscheinungen ausführbar. Diese veranlassten uns, auf Versuche über "Vagusspaltung“ nach Steiner (d u Bois' Areh. 1878) ganz zu verzichten. 
Muskeln in Thätigkeit gerathen, bis schliesslich eine Zusammenziehung der Rippenmuskeln sichtbar ist, welche länger dauert als in der Norm, und wie Timofeeff (s. unten) richtig beobachtet bat, wellenartig über den Thorax zu laufen scheint. Dass die Athempause bei vagotomirten Hunden und Katzen keine vollständige (,absolute") ist, sondern Druckänderungen in derselben stattfinden, gibt auch Schiff ${ }^{1}$ ) an; indessen fand er gegen Ende der Pause nicht eine allmähliche, durch die beginnende Inspiration veranlasste Druckverminderung, sondern im Gegentheil eine mehr oder weniger plötzliche exspiratorische Druck s te i g e r u n g durch aktive Exspiration. Einen derartigen (von Langendorff als zweizeitige, ,s akkadirte" Exspiration bezeichneten) Typus haben auch wir in seltenen Fällen bei allen drei untersuchten Thierarten beobachtet. Das Gad'sche Verfahren reizloser Durchtrennung hat Schiff nicht angewendet, indem er bei der Ausführung desselben am $\mathrm{H}$ u $\mathrm{n} d \mathrm{e}$ besondere Schwierigkeiten erwarten zu müssen glaubte. Da bei der Dicke der Nerven dieses Thieres die abgekühlten Drähte, sowie auch von Kältemischung durchflossene Röhrehen mir in der That kein genügend präzises Resultat ergaben, so nahm ich die Abliuhlung durch Auflegen der Nerven auf passend geformte Eisstückchen vor. Die Registrirung der Athmung erfolgte in diesen Versuchen durch eine mit der endständigen 'Trachealkanïle unter Einschaltung der Bert'schen Flasche verbundene Marey'sche Schreibkapsel, nachdem Vorversuche gezeigt hatten, dass dies, mangels eines Volumschreibers von genügenden Dimensionen angewendete Verfahren bei den anderen Thieren die charakteristischen Athemveränderangen nach Vagusausschaltung genïgend deutlich anzeigt. Die am Hunde mit denselben gewonnenen Kurven lassen den all mählichen Beginn und die tetanische Verlängerung der inspiratorischen $\mathrm{Ph}$ ase deutlich erkennen, während eine $\mathrm{Ver-}$ t i ef ung der Einatbmung (also gleichzeitige verstärkte Thätigkeit der Einathmungsmaskeln) n i c h t i m m er a u s g e s p r o c h en ist. Dagegen ist der exspiratorische Sehenkel der Kurve steil und die exspiratorische Zacke übersteigtdas normale Niveau-aktive Exspiration-; auf dieselbe folgt dann die, gegen die Norm mehr oder weniger

1) a. a. 0. S. 67. 
verlängertẹ exspiratoris ehe Pause, wèlche ganz allmäblich in die inspiratorische Phase iibergeht (Fig. 30, $a-c$ ).

Der Vergleich mit der normalen Athemkurve, welcher zeigt, dass die Verlangsamung der Athmung durch Verspätung sowohlder A usathmung, als a ch der Einathmung zu Stande kommt, beweist hiermit, dass auch beim Hunde sowohl hemmende als anrégende Einflüsse, welche bei der normalen Athmung betheiligt sind, $\mathrm{durch} d \mathrm{i} e \mathrm{~A}$ u s s cha $1-$ tung der. Vagi in W e g f a 11 kommen; dabei erfolgt die (verspätete) Ausathmung unter aktiver Mitwirkung der Exspirationsmuskeln, welche hier regelmässig eintritt, während sie nach unsern Erfahrungen sowohl, wie nach den Angaben anderer Autoren ${ }^{1}$ ) a $\mathrm{ch}$ bei normalen Hunden, besonders in Narkose, nicht selten ist; die Begünstigung derselben durch die beiderseitige Vagusdurchtrennung liesse sich auffassen als eine Art Ersatz für die ausgefall ène Hemmung der Inspiration durch die Lungendehnung, unter Vermittlung der Vagi, auch ohne über das Zustandekommen der aktiven Exispiration etwas Bestimmites aussagen zu können.

Was das Gesammtresultat unserer Vagusausschaltungsversuche betrifft, so sehen wir, dass die beiden Effekte, welche wir durch die zwei Hạuptarten künstlicher - elektrischer oder nicht elektrischer - Reizưng, nämlich momentane und Dauer-Einwirkung, hevorrufen konnten. - Anregung und Hemmung inspiratorischer Athembewegungen -, auch bei der Regulirung der normalen Athmung durch die Nn. vagi im Sinne der Hering-Breuer'schen Theorie betheiligt sind, und zwar bei allen untersuchten Thier. arten beide Momente nebeneinander; wie indessen je nach Art, Individualität und Zustand des Thieres bald der inspiratorische, bald der hemmende Effekt durch künstliche Einwirkungen leichter zu erzielen war, so sehen wir auch, dass je nach den genannten Umständen die Betheiligung dieser beiden Momente an der Regulirung der normalen Athmung eine verschiedene ist, und dass dasselbe für die Mitwirkung der aktiven Exspiration gilt.

Auf die Frage, warum nach beiderseitiger Vagusausschaltung die Athmung noch rhythmisch bleibt, gehen wir darum nicht näher ein, weil, wie schon oben erwähnt, wir auf die Ausdehnung unserer

1) Wertheimer, Brown-Séquard. 
Arbeit auf die Athemcentren verzichtet haben. Wir berübren diesen Punkt hier nur deshalb, weil zum Beweise dafür, dass die rhythmische Thätigkeit der Athemeentren reflektorisch und nicht automatisch erregt sei, Sch if $f^{1}$ ) angiebt, dass bei langer Lebensdauer beiderseitig vagotomirter Hunde die $R$ es $p$ ir a ti on $s$ fre q u e $\mathrm{n}$ wieder ansteige, ja dass ihr Durchschnitt schliesslich dienormale Mittelzahl wiedererreiche, was er dadurch erklärt, dass andere, für gewöhnlich an der Regulirung der Athmung nur nebenbei betheiligte centripetale Nerven, allmählich an Stelle der durchtrennten Vagi deren Funktion als Hauptregulirungsnerven der Athmung übernehmen.

Wochen-und monatelanges Ueberleben von Hunden nach beiderseitiger Vagusdurebschnei$\mathrm{d} u \mathrm{ug}$, wie es früher vielfach (durch $\mathrm{Nasse}, \mathrm{Bidder}$ und Löwins ohn, auch im hiesigen Institut) beobachtet worden ist, fand bei den erwähnten Versuchen niemals statt, indem die Thiere binnen vier Tagen der Lungenentzündung erlagen. Ich versuchte daher diejenige Abänderung des in Rede stehenden Experiments, auf welche sich jene Angabe von Schiff hauptsächlich bezieht, nämlich die, von $\mathrm{R}$ e id zuerst vorgescblagene, von $\mathrm{G}$ e $\mathrm{nz}$ e r und Fre y am Kaninchen ausgeführte, auf den Hund ausser von $\mathrm{Seh}$ iff und $\mathrm{H}$ erzen noch von $\mathrm{T}$ im ofe eff ${ }^{2}$ ) angewendete Methode: Durchschneidung des rechten Vagus unterhalb der Abzweigung des N. laryngeus recurrens, und des linken Vagus oben am Halse. Zwischen beiden Operationen liessen wir, wie Schiff und Timof eeff, einige Tage verstreichen. Die Veränderungen der Athmung waren die gleichen wie bei beiderseitiger hoher Vagusdurchschneidung; und in den zwei bisher gelungenen Versuchen blieb die Lungenentzündung aus. Leider bewirkten indessen verschiedene Umstände (junge Thiere, strenger Winter, grosse Neigung zu Eiterungen trotz umfänglicher antiseptischer Maassnahmen), dass die Lebensdauer nicht die Länge erreichte, welche nach $\mathrm{S}$ c h if $\mathrm{f}$ zur Wiederherstellung der normalen Frequenz nothwendig ist, nämlich. 14. Tage bis 3 Wochen, ein Zeitraum, welcher, wie S c h if f angiebt, und wie auch aus einer neueren Arbeit von $\mathrm{Va} n 1 \mathrm{a}$ i ${ }^{3}$ )

1) a. a. O. S. $65,69$.

2) Botkins russ. klin. Wochschr. 1889.

3) Bullet. de l'ac. roy. de Belg. (3) XXV, S. 240. 
folgt, allerdings die Regeneration der Nerven noch ausschliesst. Indessen mitissen wir unser abschliessendes Urtheil in dieser Frage aufschieben, bis uns noch mehr, gelegentlich anzustellende Versuche der in Rede stehenden Art zu Gebote stehen.

Die bisher angestellten Versuche boten uns reichlich Gelegenheit zur Untersuchung der auf die beiderseitige Vagusdurchtrennung folgenden Lungenveränderungen, über deren Natur noch keine volle Uebereinstimmung unter den Autoren besteht. Gegenüber der Tra u b e'schen, in nenerer Zeit besonders durch die ausführliche Arbeit von 0 . Fre ${ }^{1}$ ) bestätigten Annahme, dass die Lungenentzündung nach beiderseitiger Vagusdurchschneidung durch das Zusammenwirken der Kehlkopflähmung mit der Schlucklähmung zu Stande komme, indem Futtertheile, und auch bei fehlender Nahrungsaufnabme $M$ undflüsigkeit in-die Lungen gelange und die Entzündung hervoriufe; hält $\mathrm{S}$ c h i f f ${ }^{2}$ ) nach wie vor an seiner Ansicht fest, dass die Ursache aller pathologischen Lungenveränderungen nach bilateraler Vagotomie in dem Wegfall einer die Lungengefässe verengernden Funktion des Vagus zu suchen sei: „neuroparalytische Hyper$\ddot{a} \mathrm{mi} \cdot \mathrm{e}^{"}$.

Die Traube'sche Theorie stïtzt sich vor allem auf die Ergebnisse von Versuchen, einerseits die Schädlichkeit der Mundflïssigkeit $\mathrm{zu}$ beweisen, andererseits die Lungenveränderungen zu verhindern durch Abhaltnng dieser Schädlichkeit auf dem Wege der räّumlichen Trenunng des Schluckapparates und des übrigen Digestionstraktus von den Athmungswegen. So fanden Traube und Frey, dass Einlegung einer Trachealkanüle beim Kaninchen das Zustandekommen der Pneumonie verhindert, indem nur diejenigen Lungenveränderungen sich zeigen, welche auch das blosse Einlegen der (endständigen) Trachealkanüle allein, ohne Vagotomie hervorruft, ein Eingriff, welcher ja nach der von Billr oth unter Traube's Leitung gemachten Beobachtung beim Kaninchen den Tod nach sich zieht. Bei Hunden geben die Anhänger Tr a u b e's an, nach beiderseitiger Vagusdurchtrennung und Absperrung des Digestions- vom Respirationstraktus gar keine oder „nur ganz geringfügige" Veränderungen gefunden zu habęn. Hierher gehört

1) Preisschrift, Leipzig, 1879.

2) a. a. 0. S. $372 \mathrm{ff}$. 
auch das Urtheil von $\mathrm{T}$ i $\mathrm{m}$ o f e e $\mathrm{f}^{1}$ ), welcher die erwähnte Methode: Durchschneidnng des rechten Vagus unterhalb des Recurrensabganges und des linken oben am Halse, in Anwendung brachte und nach dem, nach 14 Tagen erfolgten Tode der Thiere die Lungen ohne pathologisehe Veränderungen gefunden haben will. Diesen Angaben steht diejenige von $\mathrm{Sch}$ iff gegenüher, welche während vieler Jahre eine grosse. Anzahl von Hunden nach diesem Plane operirt za baben angiebt und bei dem, oft erst nach Monaten erfolgten Tode $\left.{ }^{2}\right)$ derselben die Lungen 1 . mebr oder weniger hyperämisch, 2. von grösserem Gewicht, als dem normalen Durehschnitt entsprochen hätte, 3. den zu ihrer Aufblasung nöthigen Druck nicht unbedeutend verstärkt fand.

Vergleichen wir mit den Angaben der Autoren, von denen ich nur die wesentlichsten Punkte in aller Kürze soeben darzustellen versucht habe, unsere Befunde, so finden wir, schon bei oberflächlicher makroskopischer Inspektion, dass die a usge s prochene Pne umonie, wie sie nach doppelseitiger Vagusdurchtrennung ohne weitere Maassregeln eintritt, b e i d e $\mathrm{n}$ behufs Aufnahme der Atemvolum- resp. Druckkurven tracheotomirten und mitendständiger Luftröhrenkan üle versehenen Thieren meistens fehlte. Genauere Besichtigung, sowie anschliessende mikroskopische Untersuchung (Härtung in Alkohol, Paraffin-Durchtränkung und -Einbettung, Färbung der Schnitte mit Carmin oder Hämatoxylin) liess eine oft starke Hyperä mie, sowie beträchtliches $R$ an $d$ e $m p h$ y s m erkennen, während entzündliche Infiltrationen um die Bronchien herum meist vermisst wurden. Dass auch Ausnahmen hiervon vorkamen, in welchen die Pneunomie nach beiderseitiger Vagusdurchschneidung trotz Trachealkanïle eingetreten war, wird nach weiter unten zu erörternden Ueberlegungen verständlich erscheinen, und zwar um so mehr, als wir derartige Befunde zweimal auch nach Durchschneidung nur eines Vagus mit Einlegung der Trachealkaniile hatten, darunter das einemal eine vollständige lobäre Hepatisation der Oberlappen beider Lungen.

Bei den beiden Hunden, welche wir bis jetzt mit Erfolg nach

1) a. a. 0.

2) Diejenigen, welche über, 4 Monate lebten, wurden gelegentlich getödtet, a. a. O. S. 403. 
der Angabe von Schiff und Tim ofeeff operirt haben, bei denen also die Kehlkopflähmung nur halbseitig war ${ }^{1}$ ), fanden wir bei der Eröffinung des Thorax nach dem Tode beide Lungen in ihrer ganzen Ausdehnung von geradezu normal erscheinender, ganz schwach röthlich-weisser Farbe. Die genauere Untersuchung ergab indessen, dass sie gleichfalls nicht vollkommen normal waren, wenigstens insofern reichlich emphysematisehe Stellen vorhanden waren. "Auch erwies sich beim Einschneiden der Gehalt an Flüssigkeit als nicht unbeträchtlich, und dieselbe war blntig gefärbt. Indessen glauben wir mit der Diagnose anf "H y p e rämie", besonders mit Riicksicht auf die Möglichkeit postmortaler Entstehung der Veränderung, vorsichtig sein zu müssen.

Dass ibrigens nach beiderseitiger Vagusdurchtrennung unter Absonderung der Athmungs- von den Verdaunngswegen auch beim Hunde die Lunge etwas, wenn anch nicht stark byperämisch gefunden wird; giebt anch Frey selbst ${ }^{2}$ ) zu. Fine andere Frage ist diejenige, wie diese Hyperämie mit der Vaguslähmung znsammenlängt. Dass es sich dabei in der That um Gefässlähmung handele, wollte Michaelson ${ }^{3}$ ) ausser durch anderes anch durch die mit Hulfe eingestochener Thermonadeln gemachte Beobachtung bewiesen haben, dass nümlich sofort nach der Durchschneidung des zweiten Vagus die Temperatur der Lungen steige. Einige von uns zur Prüfung dieser Angabe vermittelst der d'Arsonval'schen Thermonadeln und eines sehr empfindlichen Multiplikators gemachte Versuche hatten kein eindeutiges Ergebniss.

Soviel erscheint indessen sicher, dass die eigentlicbe Lungenentzündung nach' doppelseitiger Vagusdurchschneidung nur dann entstehen kann, wenn die offenstehende Stimmritze das Eindringen von „Fremdkörpern" erleichtert. Dabei können immerhin die Angaben der Gegner der. Tra u be'schen Ansicht, dass durch absichtliche Einführung von Mundflüssigkeit, Futtertheilen u. s. w. in die Athmungswege nicht vagotomirter Thiere nur schwierig Pneunonie erzeugt werde, dahin gedentet werden, dass durch die Lähmung:

1) Die Thiere konnten sogar (wenn auch heisere) Laute ron sich geben, nachdem die erste Operation allein (Durchschneidung des rechten Vagus unterhalb des Recurrens) gar keinen Einfluss auf die Stimme gehabt hatte.

2) a. a. 0., S. 135.

3) Mittheilungen aus dem Königsb. physiolog. Labor., S. 85-119.

E. Pflüger, Archiv f. Physiologie Ba. 61. 
der Lungenvagi, sei es nun auf dem Wege der Bronchialmuskellähmung, oder durch ,,neuroparalytische Hyperämie", oder endlich durch Ausfall trophischer Funktionen ${ }^{1}$ ), ein Faktor gebildet werde, welcher das Entstehen der Entzindung be gio $\mathrm{n} \mathrm{s} \mathrm{t}$ i g t.

In dieser Hinsicht möchte ich daran erinnern, dass die Streitfrage über die Ursache der sogenannten Vaguspneumonie zu einer Zeit aufkam, und auch relativ neuere Arbeiten ïber diesen Gegenstand ihr noch angehören, wo die Bedeutung der $\mathrm{Mikr} 00 \mathrm{rga-}$ $\mathrm{n}$ is me $\mathrm{n}$ als Krankheitsserreger noch unbekannt war. Dass die Funktionsstörung des Lungenvagus, und wäre es auch nur durch die veränderte Athemform, die Resistenzfähigkeit der Lunge gegen Infektion vermindern könnte, ist wahrscheinlich; dass die Mundflüssigkeit, sowie Futterstoffe entründungserregende Mikroorganismen enthalten können, ist hentzutage bewiesen; auf diese Weise erscheint die Lungenentzündung nach doppelseitiger Vagusdurehschneidung wesentlich als Produkt zweier Faktoren und der Streit iiber ihre „Ursache“ als ziemlich gegenstandslos ${ }^{2}$ ).

Durch das Zusammenwirken mechanischer und thermischer Läsion (Verletzung und Erkältung) cinerseits und bakterieller Infektion andererseits dürften auch die Fälle von Pneumonie bei Kaninchen mit Trachealkaniile, aber ohne doppelseitige Vagotomie, genügend erklärt sein.

Endlich glaube ich hinsichtlich der Ur s a e he des T odes nach beiderseitiger Vagusdurchschneidung ebenso wie $\mathrm{Sigm}$. M a y e ${ }^{3}$ ), dass man in denjenigen Fällen, wo die Lungenentzündung ausbleibt, nicht nöthig hat, mit 0 . Frey die Todesursache in einem „uns noch unbekannten, dunkeln Moment" zu suchen. Aussę der von Eichhorst (Vogel), Michaelson und andern angenommenen Degeneration des Herzmuskels, derUeberanstrengung (G a d) der Athemmuskulatur, welche zu deren Paralyse führen könnte, ist vor allem an die Ernährungstörung zu

1) Vergl. hierüber Durdufi, Centralbl. f. allg. Pathol. und path. An., V, Nr. 12.

2) Eine bakteriologische Untersuchung von Lungen mit Vaguspneumonie ist neuerdings durch Lustig (Morgagni 1888) und Piccinino (Annali di nevrologia XI, 1894) vorgenommen worden. Wir haben auf eine solche zunächst verzichtet.

3) Hermann's Handb. II, 1, S, 273. 
denken, welche sehr tiefgreifend sein muss, da der Vagusstamm ausser den Athmungsorganen, dem Herzen und der Speiseröhre noch Magen ${ }^{1}$ ), Leber, Pankreas u. s. w. mit Zweigen versieht. Wie $\mathrm{T}$ i m of e e $\mathrm{f}$, welcher den Tod seiner Versuchsthiere lediglich auf Inanition zuriuckführt, sahen auch wir unsere nach seiner Methode operirten Thiere beständig erbrechen und äusserst stark abmagern. Auch konnten wir die Abnahme der Körpertemperatur konstatiren; so betrug diese bei dem in Rede stehenden Thiere, im Rectum gemessen, unmittelbar nach der zweiten Operation $40,0^{\circ}$, am sechsten Tage, ungefähr 24 Stunden vor dem Tode $35,2^{\circ}$. Eine durch besondere Versuche zu stiitzende Erklärung dieser Erscheinung fehlt noch. Ebenso wäre angesichts einer neuerdings gemachten Angabe von Morat und Dufourt ${ }^{2}$ ), dass Reizung des Vagus die Zuckerbildung in der Leber henmen kann, eine interessante, noch zu lösende Aufgabe, genau zu untersuchen, ob vagotomirte Thicre diabetisch werden.

Jedenfalls möchte ich mich der Ansicht von Sigm. Mayer anschliessen, dass bei der grossen Zahl und Wichtigkeit der physiologischen Funktionen des Vagus es eher merkwürdig wäre, wenn seine beiderseitige Durchtrennung $\mathrm{n}$ i $\mathrm{ch} \mathbf{t}$ tödtlich wäre.

1) Nach vollständigem Abschluss dieser Arbeit erschien die Veröffentlichung von $\mathrm{Paw}$ low und Schumowa (du Bois' Arch., 1895, S. 53 ff.) über die Abhängigkeit der Magensaftsecretion vom Vagus. Das von ihnen beobachtete Verhalten der rechts tief, links hoch vagotomirten Hunde stimmt mit unsern Beobachtungen gut überein.

2) Archives de physiologie (5) VI, p. $693 \mathrm{ff}$. 


\section{Erläuterung der Curven anf Tafel I, II und 11 .}

Alle diejenigen Versuche, bei welchen über Narkose nichts besonders bemerkt ist, beziehen sich auf nicht narkotisirte Thiere.

Fig. 1, 2a, 2b. Junge Katze, Registrirung der Athmung mit dem Volumschreiber. Linker Vagus intakt.

Fig. 1: Rechter Vagus undurchschnitten mechanisch tetanisirt. (Der Versuch wurde mit gleichem Erfolge an einer andern, weiter centralwärts gelegenen Stelle des Nerven wiederholt.)

Fig. 2a. Derselbe Nerv weiter centralwärts mit scharfer Scheere rasch und präzis durchschnitten.

Der centrale Stumpf wird in eine Schlinge gefasst und dieselbe - Fig. $2 b$ - fest angezogen.

Fig. 3. Kaninchen, Athemvolumschreiber. Rechter Vagus intakt; linker Vagus präzis durchschnitten.

Fig. $4 a$. Ka ninchen, A themvolumschreiber. LinkerVagus intakt; rechter durchschnitten, centraler Stumpf desselben durchströmt mit konstantem Strom aufsteigend $(\uparrow) 2$ Grenet-Elemente, $2000 \Omega$ in Nebenschliessung.

Fig. 4b. Anderes Kaninchen, oben Zwerchfell-, unten Athemvolumkurve, sonst die gleiche Versuchsanordnung, wie Fig. $4 a$.

Fig. 4c. Kaninchen, beiderseits vagotomirt; oben Thorax-, unten Volumkurve; rechter centr. Stumpf aufsteigend konstant durchströmt. 2 Grenet, $3000 \Omega$ N.-S.

Fig. 5. Hund, Morphiumnarkose, Registrirung der Athmung durch endständige 'Trachealkanüle, Bert'sche Flasche und Marey'sche Kapsel. Rechter Vagus intakt; linker a ufst. konstant durchströmt. 6 Grenet, $3000 \Omega$ N.-Schl.

Fig. 6. Katze, oben Thorax-; unten Volumkurve. Linker Vagus intakt, rechter centraler Stumpf aufsteigend konstant durchströmt, 6 Grenet, $3000 \Omega$ N.Schl.

Fig. 7. Derselbe Strom absteigend applizirt $(\downarrow)$.

Fig. 8. Kanincben, beiderseits vagotomirt, rechter centraler Stumpf aufsteigend konst. durchströmt. 4 Grenet, $10000 \Omega$ N.-Schl.

Fig. 9. Katze, Athemvolumschreiber, linker $\dot{V}$ agus intakt, rechter centraler Stumpf aufsteigend konstant durchströmt. 4 Grenet, $5000 \Omega$ N.-Schl.

Fig. 10. Kaninchen von $4 c$; gleiche Versuchsanordnung wie dort. Fig. 7 bis 10 demonstriren die entgegengesetzten Nachwirkungen.

Fig. $11 a$ und $11 b$. Dasselbe, doppelt vagotomirte Kaninchen wie $4 c$ und 10; am rechten centralen Stumpf schnell unterbrochene Kettenströme, in Fig. $11 a$ absteigend, in Fig. $11 b$ aufsteigend gerichtet. 6 Grenet, $5000 \Omega$ Nebenschliessung. 
Fig. 12. Kaninchen von 4a. Centraler Stumpf des rechten Vagus mit schnell unterbrochenen Strömen gereizt, erst auf-, dann absteigend. 2 Grenet, $2000 \Omega$ N.-S.

Fig. 13. Anderes $\mathrm{K}$ aninchen, linker Vagus intakt; centraler Stumpf des rechten mit schnell unterbrochenen Kettenströmen (60 in der Sekunde) gereizt. 6 Grenet, $2000 \Omega$ N.-S. Volumschreiber.

Fig. 14. Anderes Kaninohen. Volumsehreiber. Beide Vagi durchschnitten. Am centralen Stumpf des rechten aufsteigend, mässig schnell u n terbrochene (10 in der Sekunde) Strö me mit langer Schliessungsdauer. 6 Grenet, $2000 \Omega$.

Fig. 15 $a$ und $b$. Anderes Kaninchen. Volumschreiber. Rechter Vagus durchsehnitten, linker undurchschnitten absteigend durchströmt, in Fig. $15 a$ konstant, $15 b$ langsam unterbrochen. 6 Grenet, $10000 \Omega$ Nebenschliessung.

Fig. 15e und 16. Anderes $K$ aninchen. Volumschreiber. Beide Vagi intakt. Rechter in Fig. $15 \mathrm{c}$ absteigend langsam unterbrochen, in Fig. 16 a ufsteig end konstant durchströmt. 6 Grenet, $3000 \Omega$ N.-Schl.

Fig. 17. Kaninchen von $15 a$ u. $b$, a f steigend langsam unterbrochene Durchströmung des intakten linken Vagus.

Fig. 18 bis 20. Katze. Volumschreiber. Beide Vagi intakt. Fig. 18 absteigend konstante Durchströmung (2 Grenet, 3000 \& N.-Schl.) des rechten. Hierauf Durchsehneidung peripher von den Elektroden und Durchströmung des centralen Stumpfes: Fig. $19 a$ aufsteigend konstant, Fig. $19 b$ aufsteigend langsam unterbrochen und Fig. $19 c$ absteigend langsam unterbrochen. Danach wird das periphere über die Elektroden herabhängende Ende in leitende Berührung mit der Wunde gebracht, welche durch einen Tropfen physiologischer Kochsalzlösung gesichert wird. Fig. 20 wieder absteigend langsam unterbrochene Durchiströmung.

Fig. 21 bis 22 . Kaninchen von Fig. $4 b$. Oben Zwerchfell- unten Volum. kurve. Sog. "Unipolare" Reizung mit "differenter" Elektrode. $21 a$ und $b$ am noch intakten rechten Vagus: $21 a$ Kathode, $21 b$ Anode, 22 am durchschnittenen Nerven, Anode, also aufsteigende Durçhströmung des centralen Stumpfes. 2 Grenet, $2000 \Omega$.

Fig. 23a. Dasselbe Kaninchen, wie $4 b$ und 21 bis 22 . Reizung des noch intakten Nerven durch die Ströme des Sinusind uk tors (100 Touren).

Eig. $23 b$ und 24. Kaninchen von Fig. 4a. Volumschreiber. Linker Vagus intakt, am centralen Stumpf des rechten in $23 b$ Ströme des Sinus. induktors, in 24 frequent weahselnde (60 per Sek.) Kettenströme. 2 Grenet, $2000 \Omega$ Nebenschl.

Fig. 25. Kaninchen. Volumschreiber. Rechter Vagus durchschnitten. Bei + wird der linke Vagus auf einen stark abgekühlten Draht (Gad'sche Thermode) gelegt. 
Fig. 26. Anderes Kaninchen. Rechter undurchschnittener Vagus liegt auf der einen, stark abgekühlten Thermode. Bei + wird der linke, gleichfalls undurchschnittene Vagus auf die andere, stark abgekühlte Thermode gelegt.

Fig. 27. Anderes Kaninchen. Volumschreiber. Rechter Vagus abgekühlt. Bei + in $a$ wird auch der linke abgekühlt. Nach einiger Zeit wird der letztere (bei $o$ in $b$ ) von der Thermode wiéder abgenommen. Dasselbe wurde 4mal mit gleichem Erfolge wiederholt.

Eig. 28. Katze. Volumschreiber. Rechter Vagus abgekühlt. Bei + wird auch der linke auf die Thermode gelegt.

Fig. 29. Andere Katze. Dieselbe Versuchsanordnung.

Fig. $30 a$ bis $30 c$. Hund in ganz schwacher Morphiumnarkose. Registrirung der Athmung durch Bert'sche Flasche und Marey'sche Kapsel. Linker Vagus auf Eis. Bei + in a wird der rechte gleichfalls auf ein Eisstück gelegt. Nach Entfernung desselben erfolgte Wiedereintritt der vorherigen Athemform (etwas gegen die Norm bei schleunigt). In $b$ und $c$ unten Druckkarve nach dem Bert'schen Verfahren, oben Thoraxkurve. Bei ++ in $b$ wurde der rechte Vagus von neuem auf Eis gelegt, bei o das Eis wieder entfernt. Das gleiche Verfahren wurde noch zweimal wiederholt, zuletzt beide Vagi peripher von den Eisstücken durchschnitten. Nach einer halben Stunde Kurve $c$.

\section{Literatur-Uebersicht $1873-1894$.}

1873.

S: Arloing et L. Tripier, Contribution à la physiologie des nerfs vagues; Archives de physiol., IV, p. 411-426; 588-601; 732-742. V, p. 157-175.

A. Genzmer, Gründe für die patholog. Lungenveränderungen nach doppelseitiger Vagusdurchschneidung; Pflüger's Arch. VIII, S. 101-121.

Ph. Knoll, Ueber Reflexe auf die Athmung bei Zufuhr einiger flüchtiger Substanzen zu den unterhalb des Kehlkopfs gelegenen Luftwegen. Ber. der Wiener Akad., math.-naturw. Kl., 3. Abth., LXVIII, S. 245-273.

1874.

Sig m. Mayer, Experimenteller Beitrag zur Lehre von den Athembewegungen. Ber. der Wiener Akad., math.-naturw. K1., 3. Abth., LXIX, S. 111-122. 
1875 .

J. Rosenthal, Bemerkungen über die Thätigkeit der automatischen Nervencentra, insbes. über die Athembewegungen; Gratulationsschrift für Gerlach; Erlangen, Besold, 1875.

1876.

J. Dreschfeld, Experimental researches on the pathology of pueumonia. Lancet 1876, J, Nr. 2.

O. Frey, Die pathologischen Lungenveränderungen nach Lähmung der Nervi vagi; Preisschrift (Zürich); Leipzig, Engelmann, 1876.

L. Gerlach, Ueber die Beziehungen der Nn. vagi zu den glatten Muskelfasern der Lunge; P flügers Arch. XIII, S. 491-508.

Th. H. Mac Gillavry, De invloed van bronchialkramp op .de ademhaling; Nederl. 'Tijdschr. v. Geneesk., 1876.

P. Guttmann, Zur Lehre von den Athembewegungen; du Bois' Archiv 1875 , S. $500-525$.

1877.

F. Falk, Zur experimentellen Pathologie des X. Gehirnnerven; Archiv f. exp. Path. und Pharmakol., VII, S. 183-192.

François-Frank, Étude sur quelques arrêts respiratoires; apnée, phénomène de Cheyne-Stokes, arrêts réflexes d'origine cardiaque; Journ. de l'anat. et de la physiol., 1877, S. 545-570.

H. Köhler, Ueber die Kompensation mechanischer Respirationsstörungen und die physiologische Bedeutung der Dyspnoe; Arch. f. experim. Path. und Pharm., VII, S. 1-44.

F. Lussana e F. Ciotto, Risultanze ottenute dal taglio dei due nervi vaghi in un cane sopravissuto per diciassette giorni; Sperimentale XL, p. 119; Gazz. med. ital., 1877, giugno.

O. Rosenbach, Studien über den N. vagus; ein Beitrag zur Lehre von den automatischen Nervencentren und den Hemmungsnerven. Berlin, Hirschwald 1877.

1878.

R. Burkard, Studien über die automatische Thätigkeit des Athemcentrums und über die Beziehungen desselben zum N. vagus und anderen Athemnerven; Pflügers Arch., XVI, S. 427-501.

Th. H. Mac Gillavry, L'influence du spasme bronchique sur la respiration; Arch. néerland. d. sc. ex. et nat. XII, p. 445-456.

P. Grützner, Ueber verschiedene Arten der Nervenerregung: I, Ueber die Finwirkung ron Wärme und Kälte auf Nerven, mit Kamm und Plotke; II. Ueber die Einwirknng konstanter elektrischer Ströme auf Nerven, und III. Ueber die chemische Reizung von Nerven, mit A lex an der; Pflüger's Arch., XVII, S. 215-254 
O. Langendorff, Der Einfluss des N. vagus und der sensibeln Nerven auf die Athmung; v. Wittich's Mitheilungen aus dem Königsb. physiolog. Labor., S. 33-67.

Michaelson, Beiträge zur Untersuchung des Einflusses beiderseitiger Vagusläbmung auf die Lungen; ibid., S. 85-119.

o. Rosenbach, Notiz über den Einfluss der Vagusreizung auf die Athmung; Pflüger's Arch., XVI, S. 502-503.

J. S teiner, Ueber partielle Nervendurchschneidung und die Ursachen der Lungenaffektion nach beiderseitiger Vagustrennung am Halse; du Bois' Arch., 1878, S. 218-245.

1879.

v. Anrep, Die Ursache des Todes nach Vagusdurchschneidung bei Vögeln; Verhandl. der Würzb. med.-physik. Ges., XIV, S. 35-43.

H. Eichhorst, Die Veränderungen der quergestreiften Muskeln bei Vögeln in Folge der Inanition; Centralbl. f. d. med. Wiss., 1879, Nr. 10.

Derselbe, "Die trophischen Beziehungen der Nn. vagi zum Herzmuskel; Berlin, Hirschwald, 1879.

L. Fredericq, Sur la théorie de l'innervation respiratoire, Brux.; Hayez, 1879, und Bull. de l'acad. roy. de Belgique (2) XLVII, Nr. 4.

Derselbe, Sur l'innervation respiratoire chez le poulpe, Compt. Rend., LXXXVIII, p. $346-47$.

o. Langend orff, Ueber d. Selbststeuerung der Athembewegungen; du Bois' Arch., 1879, Suppl.-Bd., S. 48-53.

R. Zander, Folgen der Vagusdurchschneidung bei Vögeln. Preisschrift (Königsberg); Pflüger's Arch. XIX, S. 263-335.

1880.

A. Bókai, Der N. vagus und die glatten Muskelzellen der Lunge; Orvosi. Hetilap, Nr. 25.

Christiani, Ueber Athmungsnerven und Athmungscentren; Vethandl. der Berl, physiolog. Ges. in d u Bois' Arch., 1880, S. 295-96.

J. Ga d, Die Regulirung der normalen Athmung; du Boi s' Arch., 1880, S. 1-32

$\mathrm{Ph}$. Knoll, Ueber Myokarditis und die übrigen Folgen der Vagussektion bei Tauben; Prager Ztschr. f. Heilk., 1880, I.

M. Marckwald und H. Kronecker, Ueber die Auslösung der Athembewegungen; Verh. der Berl. physiolog. Ges. du Bois' Arch., 1880, S. 441-446.

J. Rosenthal, Neue Studien über Athembewegungen, 1. Die Wirkung der elektrischen Vagusreizung auf die Athembewegungen I; du Bois' Aroh., 1880, Suppl.-Bd., S. 34-49.

J. Wagner, Beiträge zur Kenntniss der respiratorischen Leistungen des N. vagus; Ber, der Wiener Akad., math.-nat. Kl, LXXX, 3. Abth., S. 177-187; Jahrb. der Gesellsch. d. Aerzte, 1880, S. $239 \mathrm{ff}$. 
1881.

A. Christiani, Ueber Athmungscentren und centripetale Athmungsnerven; Monatsberichte der Berl. Akad., 1881, S. 213-223.

J. Gad, Ueber die Abhängigkeit der Athemanstrengung vom $\mathrm{N}$. vagus; $\mathrm{du}$ Bois' Arch., 1881, S. 538-552.

M. Kandarazki, Ueber den Husten nebst einigen Bemerkungen über den Einfluss des Chloroforms auf die A thmung der Thiere; Pflïg er's Arch., XXVI, S. $470-479$.

J. Rosenthal, Neue Studien üluer Athembewegungen. 2. Ueber die Wirkung der elektr. Reizung des N. vagus II; du Bois' Arch., 1881, S. $39-60$.

N: Wedenskii, Ueber den Einfluss elektrischer Vagusreizung auf die Athembewegungen bei Säugethieren; Pflüger's Arch, XXVII, S. 1-22.

1882.

M. Ellenberger, Die Folgen der einseitigen und doppelseitigen Lähmung des N. vagus bei Wiederkäuern; Arch. für Thierheilkunde, IX, S. 128-147.

F. Henrijean, Sur les effets respiratoires de l'excitation du pneumogastrique; Archives de biologie, III, p. 229-234.

Ph. Knoll, Beiträge zur Lehre von der Athmungsinnervation, 1-3. Mittheilung; Ber. der Wiener Akad, math.-nat. Kl., 3. Abth., LXXXV, S. 282-306, LXXXVI, S. 48--65, 101-120.

S. Meltzer, Geschlecht und Lungenvagus; Centralbl. f. d. med. Wiss., 1882, S. $497-98$.

1883.

L. Fredericq, Expériences sur l'innervation respiratoire; du Bo is' Arch., 1883, Suppl.-Bd., S. $51-88$.

Ph. Knoll, Beiträge zur Lehre von der Athmungsinnervation, 4. Mittheilung; Sitzungsber. der Wiener Akad., math.-nat. Kl., 3. Abth., LXXXVIII, S. 479-512.

Laffont, Recherches sur l'innervation respiratoire; modifications des mouvements respiratoires sous l'influence de l'anesthésie; Comptes Rend., XCVII, S. $578-81$.

1884.

W. v. Anrep und N. Cybulski, Physiologische Untersuchungen im Gebiete der Athmung u. s. w., I; Petersburg 1884.

1885.

L. Fredericq, Excitation du pneumogastrique chez le lapin empoisonné par $\mathrm{CO}_{2}$; Archives de biologie, V, p. 573-580.

R. Massalongo, Contribution à l'étude expérimentale de la pneumonie et de la bronchopneumonie. Arch, de physiol. (4) II, p. 526-552. 
C. S. Roy and Gr. Brown, On bronchial contraction; Journ. of physiology, VI, proceedings phys. soc., p. XXI-XXV.

1886.

Ph. Knoll, Ueber die Athmungsinnervation; Verhandl, des Kongr. f. inn. Medicin, 1886, S. 210-227.

M. Marckwald, Die Athembewegungen und deren Innervation beim Kaninchen; Zeitschr. f. Biologie, XXIII, S. 149-284.

1887.

A. Loewy, Ueber das Athmungscentrum in der Medulla oblongata und die Bedingungen seiner Thätigkeit; du Boiș' Arch, 1887, S. 472 (Verhandl. d. Berl. physiol. Ges.).

1888.

A. Loewy, Ueber den Tonus des Lungenvagus; Pflüger's Arch., XLII, S. $273-281$.

A. Lustig, Importanza dei microorganismi nella pneumonite per vagotomia, Morgagni 1888, I, p. 392.

A. Stefani e E. Sighicelli, In qual modo il vago polmonare modifica il ritmo del respiro quando aumenta e quando diminuisce la pressione nella cavità del polmone; Sperimentale 1888, und En quelle manière etc.; Arch. italiennes de biol, XI, p. 143-163.

1889.

E. Couvreur, Influence de l'excitation du pneumogastrique sur la circulation pulmonaire de la grenouille; Comptes Rend., CIX, p. 823, Sur l'innervation vasomotrice du poumon; C. R. Soc. de biol., 1889, p. 731.

H. Head, On the regulation of respiration; Journ. of physiol., X, p. 1-70 $279-290$.

G. Heinricius, Ueber die Bedeutung der Lungenvagi bei Neugeborenen; Zeitschrift für Biologie, XXVI, S. 137-185.

Laulanié, Sur les effets respiratoires et les troubles mortels amenés par les excitations centrifuges du nerf vague; C. R. Soc. de Biol., 1889, p. 94.

A. Timofeeff, Ueber chronische Veränderungen der Athmung nach Durchschneidung beider Nn. vagi; Botkin's klin. Wochensehr., 1889, Nr. 26, 27.

1890.

Arthaud et Butte, Aetion vasomotrice du nerf pneumogastrique sur le poumon; C. R. Soc. de Biol., 1890, p. 12.

W. Cowl, The factors of the respiratory rhythm and the regulation of respiration; New-York medical Journ., 1890, 6 sept.

J. Gad, Ueber Athemreflexe von den Hauptbronchien, nach Versuchen von Zagari; Verh. d. Berl. physiol. Ges. in du Bois' Arch. 1890, S. 588- 92. 
S. J. Meltzer, On regulation of respiration; New-York medical Journ. 1890, 18. jan. Some remarks etc., ibid. 22. nov.

G. Sandmann, Zur Physiologie der Bronchialmuskulatur; du Bois' Arch., 1890, S. $252-59$.

Cather. Schipiloff, Recherches sur l'influence de la sensibilité générale sur quelques fonctions de l'organisme; Arch. des sc. phys. et nat. (3) XXIV, p. $149-175,266-284$.

1891.

Consiglio, Sur l'excitation du moignon central du vague à la suite de l'arrachement du nerf spinal ; Archives ital. de biol., 1891, XVI, p. IX.

G. Corin, Contribution à l'étude des fonctions respiratoires du nerf vague; Bull, de l'ac. roy. de Belg. (3) XXII, 516-520.

W, Einthoven, Ueber die Wirkung der Bronchialmuskeln, nach einer neuen Methode untersucht, und über Asthma nervosum; Pflüger's Arch., LI, S. $367-445$.

J. Lazarus, Ueber Reflexe von der Nasenschleimhat auf die BronchialIumina; du Bois' Arch, 1891, S. 19-36.

Fr. Spalitta, Influenza del vago e del simpatico sopra i movimenti della respirazione; Sicilia med, febbr. 1891; and Influence etc., Arcbives ital. de bio]., XV, p. $376-387$.

J. Zagari, Wirkung des Chloroforms u. s. w.; du Bois' Arch., 1891, S. 37-53;

1892.

Th. Beer, Ueber den Einfluss der peripheren Vagusreizung anf die Lunge. Centralbl. f. Physiologie V, S. 782-784; du Bois' Arch., 1892, Suppl.Bd., S. 101-216.

M. Consiglio; Sur les fibres d' arrêt de la respiration dans le tronc du vague. Arch. ital. de biol., XVII, p. 49-54.

L. Krehl, Ueber die Folgen der Vagusdurchschneidung; du Bois' Arch., 1892, Suppl.-Bd., S. 278-290.

E. Lindhagen, Ueber den Einfluss der Ausschaltung der Nn. vagi auf die Athmung beim Kaninchen; Skand. Arch. f. Physiol,, IV, S. 296-330.

S. J. Meltzer, Die athemhemmenden und -anregenden Nervenfasern innerhalb des Vagus in ihren Beziehungen zu einander und zum Athemmechanimus; du Bois' Arch., 1892, S. 340-408.

Fr. $\dot{T}_{\text {a }}$ usk, Ueber den Einfluss der Lungenvagusfasern auf den Mechanismus der Athmung. Ungar. Arch. f. Med., I, S. 397-404.

1893.

M. Doyon, Contribution à l'étude des effets circulatoires et respiratoires des excitations centrifuges du nerf vague. Archives de physiol. (5) V, p. 93.

Herzen, Des effets de la paralysie des nerfs vagues; (Soc. helvét.) Archives des sc. phys. et nat. (3) XXX, p. 626-629. 
76 Hein r. B orutta a : Untersuchungen über den Lungenvagus.

C. Vanlair, Survie après la section des deux nerfs vagues; Comptes Rend., CXVI, p. 330-331; Survie après la division successive des deux vagues; Bullet. de l'ac. roy. de Belgique (3) XXV, p. 240-260.

\section{4.}

R. Arnheim, Beiträge zur Theorie der Athmung; du Bois' Arch., 1894, S. $1 \mathrm{ff}$.

Biscons et Mouret, Note sur les effets de l'excitation du bout central du vague après arrachement du spinal; C. R. Soc. de biologie, 1894, p. 771.

F. Kauders, Ueber den Einfluss der elektrischen Reizung der Nn. vagi auf die Athmung; Pflüger's Arch. LVII, S. 333-374.

O. Langendorff und R. Oldag, Untersuchungen über das Verhalten der die A.thmung beeinflussenden Vagusfasern gegen Kettenströme; $\mathrm{P} f \mathrm{l}$ ü g e r's Arch. LIX, S. 201-224.

E. Meyer, Sur l'innervation respiratoire et l'excitation des nerfs et des muscles chez le nouveau-né; Archives de physiol. (5) VI, p. 472.

F. Piccinino, Sulla genesi della cosidetta pneumonite del vago; Ann. di neurol. XI; Neurol. Centralbl. XIII, S. 556. 